\title{
AN ELASTO-VISCOPLASTIC CONSTITUTIVE MODEL WITH STRAIN-SOFTENING FOR SOFT SEDIMENTARY ROCKS
}

\author{
TOSHIHISA AdACHI ${ }^{\mathrm{i})}$, Fusao OKA ${ }^{\mathrm{ii})}$ and MASASHI KoIKE ${ }^{\mathrm{iii})}$
}

\begin{abstract}
In general, soft sedimentary rocks exhibit strain-softening and time-dependent behavior. In the present paper, an elasto-viscoplastic constitutive model for soft rocks is proposed, that can universally describe time-dependent behavior such as creep, stress relaxation and strain rate sensitivity by extending Adachi and Oka's elasto-viscoplastic model for frozen sand. It is found that the proposed model can be applied to the time-dependent behavior of a soft tuffaceous rock called Tomuro Stone under the strain rate constant triaxial compression, drained triaxial creep and undrained triaxial stress relaxation.
\end{abstract}

Key words: constitutive model, elasto-viscoplasticity, soft rock, strain softening, time dependency (IGC: F6)

\section{INTRODUCTION}

The aim of the present paper is to derive a constitutive model for soft rocks. It is well known that soft rocks exhibit strain softening and time dependent behavior (Adachi and Ogawa, 1981; Elliot and Brown, 1985; Aversa et al., 1993 etc.). "Strain softening" refers to the phenomenon that the stress increases with the increase of strain until the peak value of stress and then decreases during straining, and finally approaches a strength at large strain or residual strength under the confining pressure which is less than the critical value of confining pressure for the materials. In the elasto-plastic model the strain softening is explained as the decrease of the yield stress. During the strain softening, positive dilatancy is observed. Strain softening is deeply related to the progressive failure of geologic materials. One of the reasons for strain softening is, deterioration of the material microstructure which is considered in the present study. Another reason is due to strain localization i.e. strain band formation with strain-hardening constitutive model. This type of approach provides the important information on the onset condition of shear band. However, for the post localization behavior we need a more sophisticated model such as a non-local model to avoid the ill-posedness of the boundary value problem (see, Mühlhaus and Aifantis, 1991; Adachi and Oka, 1995). In addition, it is worth noting that the strain softening due to deterioration of the material provides the description of the strain localization.

The structure of soft rocks and stiff soils gradually deteriorates during strain process. In the formulation of soft rocks, this destructuration process has to be modeled. Many researchers have been developing constitutive models considering the destructuration or debonding (e.g., Nova, 1992; Gens and Nova, 1993; Kavvadas, 1993; Nova et al., 2003). On the other hand, the behavior of soft rocks greatly depends on the strain rate and/or duration time of loading which is called the time dependent behavior. Time dependency refers to the phenomenon that mechanical response of a material depends on the time under the external application of stress, such as creep, stress relaxation and strain rate sensitivity. These phenomena are not independent of each other but different aspects of time dependent nature of materials (Akai et al., 1974, 1975). The time dependency is the salient feature of fracture and the strength deterioration of geologic materials (Adachi et al., 1996). Since soft rocks are the materials that strongly exhibit strain softening and time dependency (Akai et al., 1977), both phenomena should be taken into account for the mechanical analysis of soft rocks. Although many researchers have proposed several constitutive models for time dependent behavior of soft rocks until now (e.g. Cristescu, 1994), only a few models have been studied that can describe both strain softening and time dependent behavior. As it has been studied by many researchers, strain softening may be the main cause of strain localization and progressive failure (Adachi et al., 1991; Oka et al., 1994; Adachi et al., 1994). Thereby, it is very important to analytically investigate the mechanical behavior of soft rocks with the models that can describe two phenomena that controls the long-term instability problems in geotechnical engineering field.

iii) Taisei Construction Co., Tokyo, Japan.

The manuscript for this paper was received for review on March 1, 2004; approved on January 18, 2005.

Written discussions on this paper should be submitted before November 1, 2005 to the Japanese Geotechnical Society, 4-38-2, Sengoku, Bunkyo-ku, Tokyo 112-0011, Japan. Upon request the closing date may be extended one month. 
In the present paper, we have proposed an elastoviscoplastic model for soft rocks that can describe both strain softening and time dependent behavior based on the elasto-viscoplastic model for frozen sand (Adachi et al., 1990, 1992). In addition, the proposed model was verified with the use of experimental findings of undrained triaxial compression test results, drained creep test and undrained stress relaxation test for Tomuro stone.

\section{DERIVATION OF ELASTO-VISCOPLASTIC CONSTITUTIVE MODEL}

\section{New Time Measure and Stress History Tensor}

Oka (1985) proposed a new method to construct an elasto-viscoplastic model based on a generalized simple body in which yield function depends on a stress history tensor. Oka's model (1985) can be continuously reduced to the inviscid model in the limiting case. Adachi and Oka $(1985,1995)$ derived a strain-softening model for geologic materials based on stress history tensor in which stress history is defined by generalized strain measure. The strain measure adopted in that model is similar to the endochronic concept advocated by Valanis (1971).

Adachi, Oka and Poorooshasb (1990) and Adachi and Oka (1992) proposed a new type of elasto-viscoplasticity model for frozen sand introducing a new time measure instead of real stress for frozen sand as

$$
d z=g\left(\dot{\varepsilon}_{\mathrm{ij}}^{*}\right) d t, \quad z=\int d z
$$

where $d z$ is the increment of a new time measure, $d t$ is the real time increment, the function $g$ is the function that describes strain rate dependency to be determined experimentally for the particular medium, and $\dot{\varepsilon}_{\mathrm{ij}}^{*}$ is the state parameter for the strain rate history which includes current strain rate and the strain rates in the loading history.

Under the three dimensional conditions, Eq. (1) can be rewritten as

$$
\begin{aligned}
& d z=g(F)^{\mathrm{a}} d t \\
& F=\hat{F}\left(I_{1}, I_{2}\right)
\end{aligned}
$$

where $d t$ is the real time increment, function $g$ is the function that describes the strain rate dependency to be determined experimentally for a particular medium, $I_{1}$ and $I_{2}$ are first and second invariant of strain rate tensor as: $I_{1}=\dot{\varepsilon}_{\mathrm{kk}}, I_{2}=\sqrt{\dot{e}_{\mathrm{ij}} \dot{e}_{\mathrm{ij}}}$ and $a$ is the material parameter for the strain rate history.

In the present paper the stress history tensor will be used for the formulation of yield function. The stress history tensor $\sigma_{i j}^{*}$ is given as a functional of reduced stress history with respect to the new time measure $z$. Here, the stress history tensor is expressed by a single exponential type kernel function as follows:

$$
\sigma_{\mathrm{ij}}^{*}=\int_{0}^{z} \frac{1}{\tau} \exp \left[\left(z-z^{\prime}\right) / \tau\right]\left[\sigma_{\mathrm{ij}}\left(z^{\prime}\right)-\sigma_{\mathrm{ij}}(0)\right] d z^{\prime}+\sigma_{\mathrm{ij}}^{*}(0)
$$

of stress with respect to the time measure, $\sigma_{\mathrm{ij}}(0)$ and $\sigma_{\mathrm{ij}}^{*}(0)$ are the values of the stress tensor and stress history tensor at $z=0$.

As it has been studied by Adachi and Oka (1995), the stress history tensor has been introduced for controlling the stress-strain behavior of frictional strength materials. Hence, the stress history tensor is used in the yield functions in the following. Let us consider the behavior of soft rock under conventional triaxial compression test conditions for explanation. The structure of soft rocks and stiff soils gradually deteriorates during strain process. This destructuralization process can be controlled by the stress history tensor. In this case, the deviator stress history $q^{*}$ ( $q$ is the deviator stress: $\sigma_{1}^{\prime}-\sigma_{3}^{\prime}, \sigma_{1}^{\prime}$ : maximal principle stress, $\sigma_{3}^{\prime}$ : minimum principal stress and $q^{*}$ is the corresponding deviator stress history) that increases with increase of strain and at large strains, the deviator stress history tensor becomes almost equal to the current stress. In addition, when $\tau=0$, the deviator stress history coincides with the real deviator stress. It means that the material with $\tau=0$ corresponds to the fully destructured materials in which internal structure formed by cementation etc. is fully removed. In the present model, Terzhagi's effective stress concept is used without notice for water saturated soft rock.

By introducing a new time measure in the elasto-plastic model with strain softening, Adachi, Oka and Poorooshasb (1990) and Adachi and Oka (1992) proposed an elasto-viscoplastic model for frozen sand and verified its applicability. This model is able to describe the strain rate dependency of materials. It was, however, found that the model was not adequate enough to accurately reproduce those time dependent behaviors such as creep and stress relaxation. In order to improve this shortcoming, it is assumed that the behavior of materials depends not only on the present strain rate but also on the average strain rate that has been experienced.

In order to extend the time measure, the function $F$ is introduced with the average strain rate tensor.

$$
\begin{aligned}
& \bar{F}=\hat{F}\left(\bar{I}_{1}, \bar{I}_{2}\right) \\
& \bar{I}_{1}=\bar{\varepsilon}_{\mathrm{kk}} / \bar{t}, \bar{I}_{2}=\sqrt{\overline{\dot{e}}_{\mathrm{ij}} \overline{\dot{e}}_{\mathrm{ij}}}, \quad \bar{\varepsilon}_{\mathrm{ij}}=\int \dot{\varepsilon}_{\mathrm{ij}} d t, \overline{\dot{\varepsilon}}_{\mathrm{ij}}=\bar{\varepsilon}_{\mathrm{ij}} / \bar{t}
\end{aligned}
$$

where $\dot{\varepsilon}_{\mathrm{ij}}$ is the strain rate tensor, $\bar{t}$ is the time measured from the start of the tests to the present time. $\bar{\varepsilon}_{\mathrm{ij}}$ is the accumulated strain during the tests, $\dot{e}_{\mathrm{ij}}$ is the deviatoric component of strain rate tensor.

Based on the extended strain rate function $\bar{F}$, an increment of a new time measure $z$ is introduced for Eq. (2) as

$$
d z=\left(C \frac{\bar{F}}{\bar{F}_{0}}+(1-C) \frac{F}{F_{0}}\right)^{\mathrm{a}} d t
$$

where $a$ and $C$ are material constants, $\bar{F}_{0}$ and $F_{0}$ are referential values of $\bar{F}$ and $F$.

Under axisymmetric triaxial conditions, Eq. (6) can be written as 


$$
d z=\left(C \frac{\overline{\dot{\varepsilon}}_{11}}{\bar{\varepsilon}_{110}}+(1-C) \frac{\dot{\varepsilon}_{11}}{\dot{\varepsilon}_{110}}\right)^{\mathrm{a}} d t=\left(C \frac{\bar{\varepsilon}_{11} / \bar{t}}{\overline{\dot{\varepsilon}}_{110}}+(1-C) \frac{\dot{\varepsilon}_{11}}{\dot{\varepsilon}_{110}}\right)^{\mathrm{a}} d t
$$

in which the subscripts 1 and 0 denote the axial component strain rate and the referential component respectively.

In the definition of Eq. (7), strain rate associated with material behavior is considered as the sum of the average strain rate and the instantaneous strain rate using a weighting parameter $C$. By applying this newly introduced time measure to Adachi and Oka's model for frozen sand, it introduces a new elasto-viscoplastic constitutive model with strain softening for soft rocks.

This new definition of time measure is reduced for some specific testing conditions, that is, axisymmetric triaxial compression test.

a) For strain rate constant conventional (axisymmetric) triaxial compression test in which $\overline{\tilde{\varepsilon}}_{11}=\dot{\varepsilon}_{11}=$ const.

$$
d z=\left(\frac{\dot{\varepsilon}_{11}}{\dot{\varepsilon}_{110}}\right)^{\mathrm{a}} d t
$$

b) For triaxial creep condition, stresses are kept constant.

$$
d z=\left(C \frac{\bar{\varepsilon}_{11}}{\dot{\varepsilon}_{110}}+(1-C) \frac{\dot{\varepsilon}_{11}}{\dot{\varepsilon}_{110}}\right)^{\mathrm{a}} d t
$$

c) For stress relaxation in which strain rate is kept 0 , that is, $\dot{\varepsilon}_{11}=0$.

$$
d z=\left(C \frac{\bar{\varepsilon}_{11} / \bar{t}}{\dot{\varepsilon}_{110}}\right)^{\mathrm{a}} d t=\left(C \frac{\bar{\varepsilon}_{110}}{\dot{\varepsilon}_{110} \bar{t}}\right)^{\mathrm{a}} d t
$$

\section{Strain Increment and Flow Rule}

Herein, only infinitesimal strain fields are addressed with and the total strain rate tensor is decomposed into its elastic and viscoplastic components, i.e.,

$$
d \varepsilon_{\mathrm{ij}}=d \varepsilon_{\mathrm{ij}}^{\mathrm{e}}+d \varepsilon_{\mathrm{ij}}^{\mathrm{p}}
$$

The elastic strain increment is given in a linear elastic form given by;

$$
d \varepsilon_{\mathrm{ij}}^{\mathrm{e}}=\frac{d s_{\mathrm{ij}}}{2 G}+\frac{d \sigma_{\mathrm{m}}^{\prime}}{3 K} \delta_{\mathrm{ij}}
$$

where $G$ is the elastic shear modulus, $K$ is the elastic bulk modulus, $s_{\mathrm{ij}}$ is the deviatoric stress component and $\sigma_{\mathrm{m}}^{\prime}$ is the mean effective stress.

The viscoplastic strain rate $d \varepsilon_{\mathrm{ij}}^{\mathrm{p}}$ is assumed to be given by the following non-associated flow rule;

$$
d \varepsilon_{\mathrm{ij}}^{\mathrm{p}}=\Lambda \frac{\partial f_{\mathrm{p}}}{\partial \sigma_{\mathrm{ij}}^{\prime}} d f_{\mathrm{y}}
$$

in which $f_{\mathrm{p}}$ is the plastic potential function, $f_{\mathrm{y}}$ is the yield function and $\Lambda$ is the plastic loading index.

\section{Plastic Yield Function}

The subsequent yield function is assumed to be a function of the stress history tensor $\sigma_{\mathrm{ij}}^{*}$ and hardening/ softening parameter $\kappa$, and is given by;

$$
\begin{aligned}
& f_{\mathrm{y}}=\eta^{*}-\kappa=0 \\
& \eta^{*}=\left(\eta_{\mathrm{ij}}^{*} \eta_{\mathrm{ij}}^{*}\right)^{1 / 2} \\
& \eta_{\mathrm{ij}}^{*}=s_{\mathrm{ij}}^{*} / \sigma_{\mathrm{m}}^{*}
\end{aligned}
$$

where $s_{i j}^{*}$ is a deviatoric part of the stress history tensor and $\sigma_{\mathrm{m}}^{*}$ is the mean stress history.

Equation (14) is a yield function for the change of stress ratio. As for the change of mean effective stress, the additional yield function, i.e. the second yield function for the change of $\sigma_{\mathrm{m}}^{\prime}$ is necessary with the second hardening parameter. However, the second yield function is not specified because the effect of the change of mean effective stress is not large in the overconsolidated region.

Next the loading conditions are specified as follows;

Loading: if $f_{\mathrm{y}}=0$ and $d f_{\mathrm{y}}=\left(\partial f_{\mathrm{y}} / \partial \sigma_{\mathrm{ij}}^{*}\right)_{\mathrm{k}=\text { const. }} d \sigma_{\mathrm{ij}}^{*}>0$, $d \varepsilon_{\mathrm{ij}}^{\mathrm{p}} \neq 0$.

Neutral loading: if $f_{\mathrm{y}}=0$ and $d f_{\mathrm{y}}=\left(\partial f_{\mathrm{y}} / \partial \sigma_{\mathrm{ij}}^{*}\right)_{\mathrm{k}=\text { const. }}=0$, $d \varepsilon_{\mathrm{ij}}^{\mathrm{p}}=0$.

Unloading: if $f_{\mathrm{y}}=0$ and $d f_{\mathrm{y}}=\left(\partial f_{\mathrm{y}} / \partial \sigma_{\mathrm{ij}}^{*}\right)_{\mathrm{k}=\text { const. }}<0$, $d \varepsilon_{\mathrm{ij}}^{\mathrm{p}}=0$.

\section{Strain Hardening-Softening Parameter}

The evolutional equation of strain hardening-softening parameter $\kappa$ is assumed to be given by the following relation;

$$
\dot{\kappa}=\frac{G^{\prime}\left(M_{\mathrm{f}}^{*}-\kappa\right)^{2} \dot{\gamma}^{\mathrm{p}}}{M_{\mathrm{f}}^{* 2}}
$$

in which $\dot{\gamma}^{\mathrm{p}}$ is the second invariant of the deviatoric plastic strain rate tensor, given by the equation;

$$
\dot{\gamma}^{\mathrm{p}}=\left(\dot{e}_{\mathrm{ij}}^{\mathrm{p}} \dot{e}_{\mathrm{ijj}}^{\mathrm{p}}\right)^{1 / 2}
$$

where $\dot{e}_{\mathrm{ij}}^{\mathrm{p}}$ is the deviatoric plastic strain rate tensor. Then $\kappa$ is given by

$$
\kappa=\int_{0}^{t} \dot{\kappa} d t
$$

When $\gamma^{\mathrm{p}}$ is zero at an initial state under proportional loading condition, the following hyperbolic function is given by the integration of Eq. (17).

$$
\kappa=\frac{M_{\mathrm{f}}^{*} G^{\prime} \gamma^{\mathrm{p}}}{M_{\mathrm{f}}^{*}+G^{\prime} \gamma^{\mathrm{p}}}
$$

in which $M_{\mathrm{f}}^{*}$ is the value of $\eta^{*}$ at the residual strength state and $G^{\prime}$ is the initial tangent of Eq. (20). Considering Eqs. (14) and (17), the material parameter $G^{\prime}$ can be determined by the initial tangent of the relationship between $\gamma^{\mathrm{p}}$ and $\kappa$.

It can be seen from Eq. (20) that the hardening-softening parameter $\kappa$ always increases with the strain. Let us consider the one-dimensional case with monotonic strain or displacement control test for simplicity. In the early straining process, the stress increases with higher rate than the parameter $\kappa$. Then, the stress increases to some extent, the viscoplastic strain rate becomes very large. Subsequently, the stress decreases with increase in strain. This is a reason why the present model can describe the strain softening process. Owing to this feature that the 


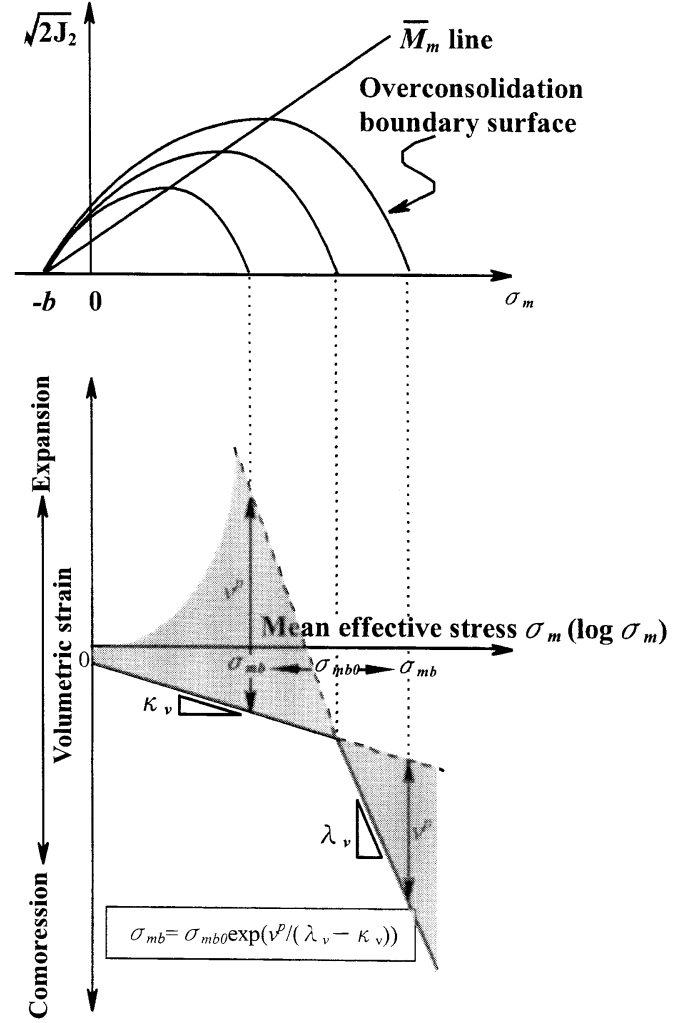

Fig. 1. Variation of $\sigma_{\mathrm{mb}}$

hardening and softening parameter always increases with non-negative sign, the present formulation does not lead to the instability problem of the conventional strain softening model with negative modulus discussed by many researchers e.g. Valanis (1985).

\section{Plastic Potential Functions and Overconsolidation Boundary Surface}

The plastic potential function is assumed to be a function of true stress and is given by the following form, which is similar to the function for soft rock.

$$
f_{\mathrm{p}}=\bar{\eta}+\bar{M} \ln \left[\left(\sigma_{\mathrm{m}}+b\right)\left(\sigma_{\mathrm{mb}}+b\right)\right]=0
$$

in which $b$ and $\sigma_{\mathrm{mb}}$ are material parameters, and $\bar{\eta}$ is the stress invariant ratio defined by;

$$
\bar{\eta}=\left[s_{\mathrm{ij}} S_{\mathrm{ij}} /\left(\sigma_{\mathrm{m}}+b\right)^{2}\right]^{1 / 2}
$$

$b$ and $\sigma_{\mathrm{mb}}$ are the parameters that describe material structures.

Furthermore, $\sigma_{\mathrm{mb}}$ depends on the plastic volumetric strain $v^{\mathrm{p}}$ as shown in Fig. 1 in which $\sqrt{2 J_{2}}\left(=\sqrt{s_{\mathrm{ij}} S_{\mathrm{ij}}}\right)$ is the second invariant of deviatoric stress tensor $s_{\mathrm{ij}}$, and is defined as;

$$
\sigma_{\mathrm{mb}}=\sigma_{\mathrm{mb} 0} \exp \left[v^{\mathrm{p}} /\left(\lambda_{\mathrm{v}}-\kappa_{\mathrm{v}}\right)\right]
$$

where $\sigma_{\mathrm{mb} 0}$ is the initial value of $\sigma_{\mathrm{mb}}$ at $v^{\mathrm{p}}=0, \lambda_{\mathrm{v}}$ is the compression index and $\kappa_{\mathrm{v}}$ is the swelling index respectively.

The overconsolidation boundary surface is also introduced and is assumed to move reflecting the change of internal structures of material.

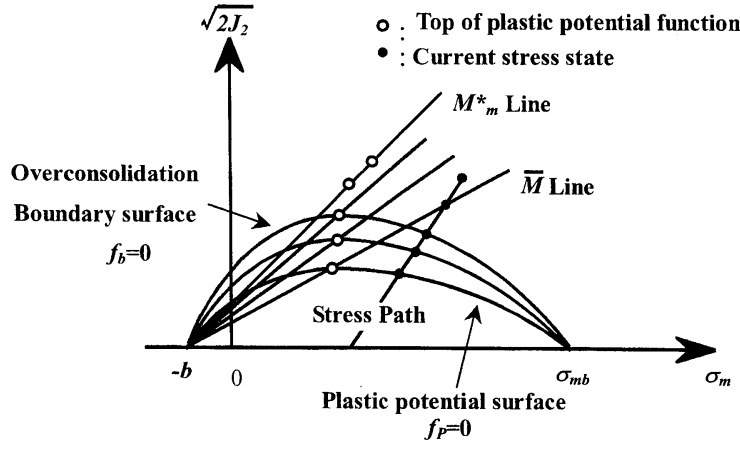

Fig. 2. Plastic potential and overconsolidation boundary surfaces

$$
f_{\mathrm{b}}=\bar{\eta}+\bar{M}_{\mathrm{m}} \ln \left[\left(\sigma_{\mathrm{m}}+b\right) /\left(\sigma_{\mathrm{mb}}+b\right)\right]=0
$$

where $\bar{M}_{\mathrm{m}}$ is the value of stress invariant ratio $\bar{\eta}$ when the maximum compressive volumetric strain takes place during shear deformation. Inside the boundary surface $\left(f_{\mathrm{b}}<0\right), \bar{M}$ is given by;

$$
\bar{M}=-\bar{\eta} / \ln \left[\left(\sigma_{\mathrm{m}}+b\right) /\left(\sigma_{\mathrm{mb}}+b\right)\right]
$$

On the other hand, outside the boundary surface $\left(f_{\mathrm{b}} \geq 0\right)$, $\bar{M}$ is constant as

$$
\bar{M}=\bar{M}_{\mathrm{m}}
$$

The boundary surface and plastic potential function in the case of isotropically consolidated state are shown in Fig. 2.

\section{Viscoplastic Strain Increment}

In the present paper, the first yield function is only used in the analysis because soft rock is in the overconsolidation surface under relatively low confining pressure.

Taking into account the Prager's consistency condition for plastic loading

$$
d f_{\mathrm{y}}=d\left(\eta^{*}-\kappa\right)=0
$$

together with Eqs. (10), (13) and (21), the viscoplastic strain increment $d \varepsilon_{\mathrm{ij}}^{\mathrm{p}}$ is given by;

$$
d \varepsilon_{\mathrm{ij}}^{\mathrm{p}}=\Lambda\left[\frac{\bar{\eta}_{\mathrm{ij}}}{\bar{\eta}}+(\bar{M}-\bar{\eta}) \frac{\delta_{\mathrm{ij}}}{3}\right]\left[\frac{\eta_{\mathrm{kl}}^{*}}{\eta^{*}}-\eta^{*} \frac{\delta_{\mathrm{kl}}}{3}\right] \frac{d \sigma_{\mathrm{kl}}^{*}}{\sigma_{\mathrm{m}}^{*}}
$$

in which

$$
\begin{aligned}
& \Lambda=\frac{M_{\mathrm{f}}^{* 2}}{G^{\prime}\left(M_{\mathrm{f}}^{*}-\kappa\right)^{2}} \\
& \bar{\eta}_{\mathrm{ij}}=s_{\mathrm{ij}} /\left(\sigma_{\mathrm{m}}+b\right) \\
& \eta_{\mathrm{kl}}^{*}=s_{\mathrm{kl}}^{*} / \sigma_{\mathrm{m}}^{*}
\end{aligned}
$$

The derived model contains the following 12 material parameters,

$G$ : elastic shear modulus,

$K$ : elastic bulk modulus,

$\lambda_{\mathrm{v}}$ : compression index of $v-\ln \sigma_{\mathrm{m}}^{\prime}$ relation,

$\kappa_{\mathrm{v}}$ : swelling index of $v-\ln \sigma_{\mathrm{m}}^{\prime}$ relation,

$b$ : plastic potential parameter,

$\sigma_{\mathrm{mb} 0}$ and $\bar{M}_{\mathrm{m}}$ : parameters for overconsolidation boundary surface, 
Table 1. Material parameters

\begin{tabular}{c|c|c|c}
\hline$\sigma_{3}^{\prime}(\mathrm{kPa})$ & 98 & 294 & 490 \\
\hline \hline$G\left(\mathrm{MN} / \mathrm{m}^{2}\right)$ & 157.3 & 195.3 & 213.4 \\
\hline$G^{\prime}\left(\mathrm{MN} / \mathrm{m}^{2}\right)$ & 65.5 & 110.8 & 164.2 \\
\hline$K\left(\mathrm{MN} / \mathrm{m}^{2}\right)$ & 135.6 & 179.9 & 194.8 \\
\hline$M_{\mathrm{f}}^{*}$ & 1.85 & 1.53 & 1.47 \\
\hline $\bar{M}_{\mathrm{m}}$ & & 1.25 & \\
\hline$\sigma_{\mathrm{mb} 0}(\mathrm{kPa})$ & & 7480 & \\
\hline$b(\mathrm{kPa})$ & & 852.6 & \\
\hline$\kappa_{\mathrm{v}}$ & & 0.00571 & 0.03 \\
\hline$\lambda_{\mathrm{v}}$ & & 0.0802 & 13800 \\
\hline$\tau$ & 53100 & 0.953 & 0.02 \\
\hline$a$ & 0.928 & & \\
\hline$C$ & 0.04 & & \\
\hline
\end{tabular}

$M_{\mathrm{f}}^{*}$ : stress invariant ratio at failure (residual strength state),

$G^{\prime}$ : strain hardening parameter,

$\tau$ : stress history parameter,

$a$ and $C$ : parameters relating to rate-dependent behavior.

\section{SIMULATION OF BEHAVIOR OF SOFT ROCK IN TRIAXIAL TEST}

In the present section, we will investigate an applicability of the proposed elasto-viscoplastic model for soft rocks by simulating the behavior of Tomuro stone (sedimentary soft rock) under the conventional triaxial conditions (axisymmetric).

\section{Determination of Parameters}

Material parameters of Tomuro stone determined from test results are listed in Table 1. The followings are the ways to determine the material parameters.

(a) Elastic shear modulus $G$ and elastic bulk modulus $K$ are to be determined by the initial tangential slopes of deviatoric stress-deviatoric strain relation and volumetric strain-deviatoric strain relation, respectively.

(b) Compression index $\lambda_{\mathrm{v}}$ and swelling index $\kappa_{\mathrm{v}}$ are to be determined by $v-\ln \sigma_{\mathrm{m}}^{\prime}$ curve obtained by isotropic consolidation and swelling tests.

(c) Plastic potential parameter $b$ and parameter $\bar{M}_{\mathrm{m}}$ for overconsolidation boundary surface can be obtained by the stress ratio $\bar{\eta}$ at the states when the maximum volumetric compression takes place during shearing process shown in Fig. 3.

(d) Parameter $\sigma_{\mathrm{mb}}$ for overconsolidation boundary surface is the consolidation yielding stress obtained by isotropic consolidation test.

(e) Parameter $M_{\mathrm{f}}^{*}$ is determined as the stress ratio at large strain state, that is, the residual strength state

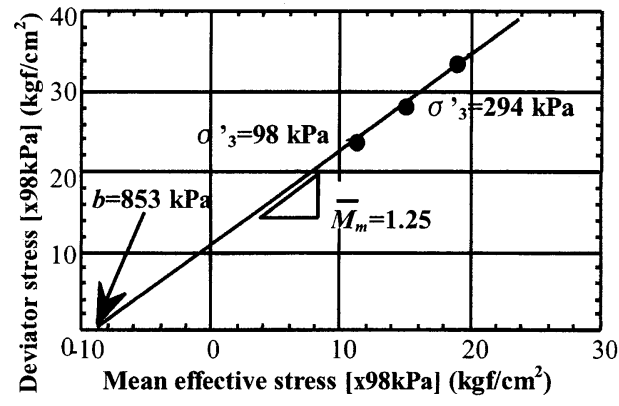

Fig. 3. Determination of parameters $b$ and $\bar{M}_{\mathrm{m}}$

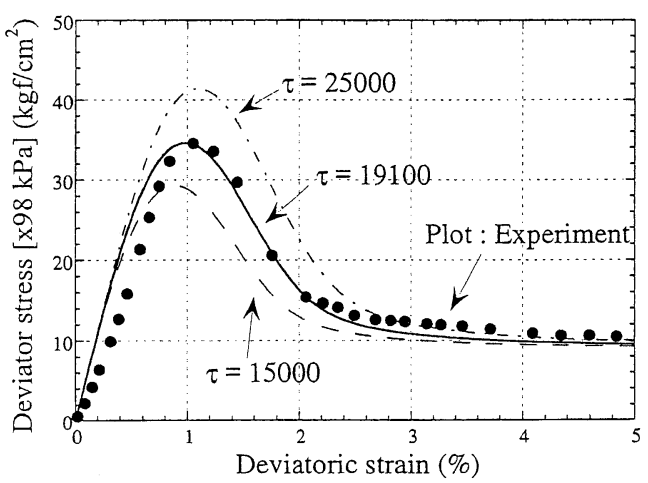

Fig. 4. Determination of $\tau^{\prime}$

and $G^{\prime}$ can be approximately determined from the stress ratio-plastic strain relationship of unloading and reloading process at large strains or more practically stress-strain curve adjusting method after the other parameters are fixed.

(f) In order to determine the stress history parameter $\tau$ and time dependent parameter $a$, at least two strain rate constant triaxial compression tests should be conducted with different strain rates under the same confining pressure. At first, by taking $a=0$, i.e., $d z=d t$, in Eq. (6), the parameter $\tau$ is determined by a process of trial and error analyses such that the best fit is obtained for the stress-strain curve for each strain rate $\dot{\varepsilon}_{11}$ as shown in Fig. 4. Thus obtained $\tau$ is denoted as $\tau^{\prime}$. Since the best fitting parameter $\tau^{\prime}$ is determined for each strain rate $\dot{\varepsilon}_{11}$, $\tau^{\prime}-\dot{\varepsilon}_{11}$ relation can be obtained as illustrated in Fig. 5. From this figure, the following relation can be obtained.

$$
\tau^{\prime}=\left(\frac{\dot{\varepsilon}_{11}}{\dot{\varepsilon}_{110}}\right)^{-\mathrm{a}} \tau_{0}, \quad a \geq 0
$$

in which $\tau_{0}$ is the value of $\tau^{\prime}$ at $\dot{\varepsilon}_{11}=\dot{\varepsilon}_{110}$. In the figure, if setting $\dot{\varepsilon}_{110}=10^{-3} \% / \mathrm{min}, \tau_{0}=4.5 \times 10^{3}$ is determined. Thus determined $\tau_{0}$ is independent of strain rate and is considered to be an intrinsic material constant. And we define that this $\tau_{0}$ is the stress history parameter $\tau$. Furthermore, the time dependent parameter $a$ can be determined as the slope of the relation. 


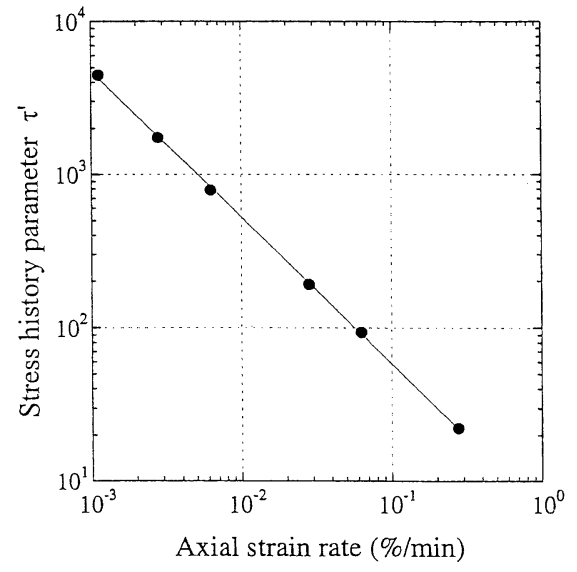

Fig. 5. Determination of $\tau^{\prime}$ and $a$

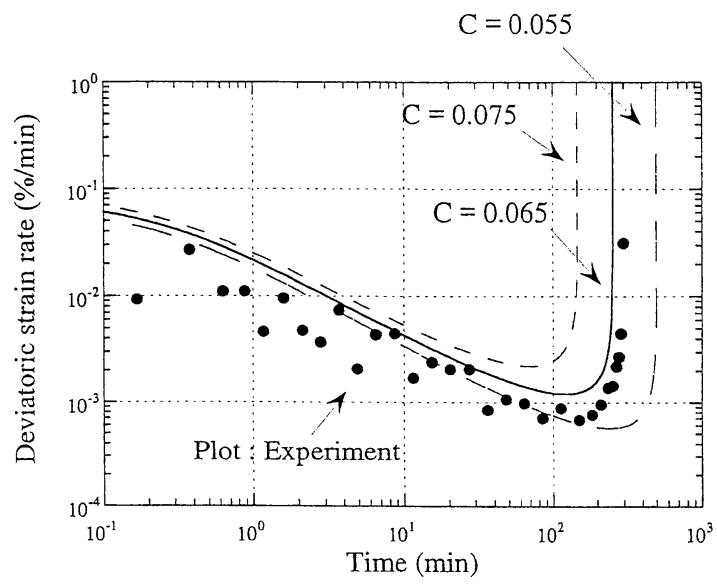

Fig. 6. Determination of $C$

(g) In order to determine the time dependent parameter $C$, creep test or stress relaxation test should be conducted. A process of trial and error analyses determine the parameter $C$ such that the best fit is obtained for the deviatoric strain rate-time curve as shown in Fig. 6. We assume that the material parameters $a$ and $C$ are constant, however, as will be seen in Table 1, they depend on the confining pressure. The confining-pressure dependency of these parameters have to be studied in future study.

\section{Application to the Results of Triaxial Tests}

The newly developed elasto-viscoplastic model was applied to the time-dependent behavior of Tomuro stone. Tomuro stone is a tuffaceous soft rock and kind of Ohya stone produced in the Tomuro district of Ohya in Tochigi prefecture Japan. A block sample, $400 \mathrm{~mm} \times 400 \mathrm{~mm} \times$ $1000 \mathrm{~mm}$ was sampled in the Tomuro district. The fundamental properties of Tomuro stone are as follows: specific gravity is 2.51 , initial void ratio is 0.63 , and the uniaxial compression strength is $3.05 \mathrm{MPa}$. Strain rate constant triaxial compression tests, drained creep tests and undrained stress relaxation tests were performed.

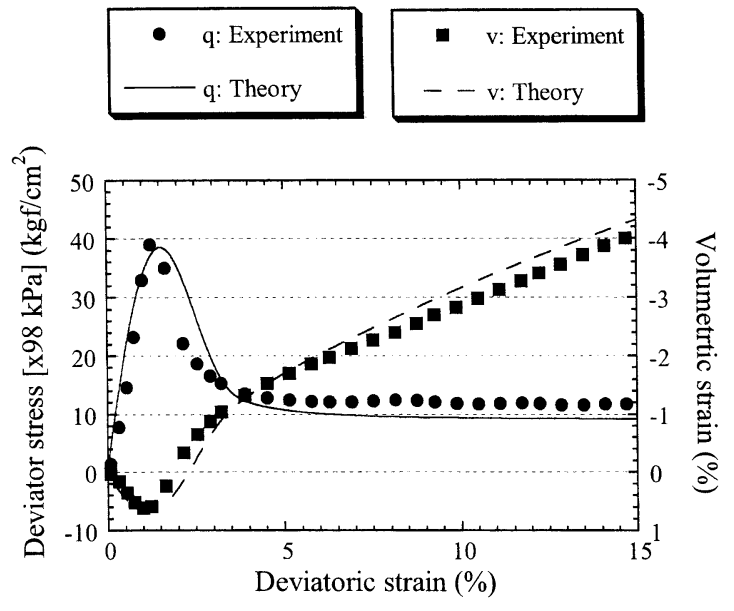

(a)

Fig. 7(a). Deviator stress-deviatoric strain and volumetric straindeviatoric strain relations (Strain rate $=\mathbf{2 . 8} \times \mathbf{1 0}^{-1} \% / \mathrm{min}$, confining pressure $98 \mathrm{kPa}$ )

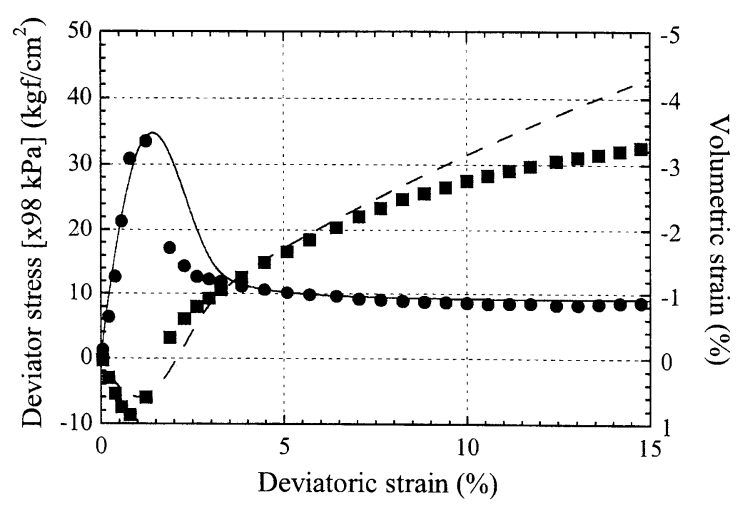

(b)

Fig. 7(b). Deviator stress-deviatoric strain and volumetric straindeviatoric strain relations (Strain rate $=\mathbf{2 . 8} \times \mathbf{1 0}^{-2} \% / \mathrm{min}$, confining pressure $98 \mathrm{kPa}$ )

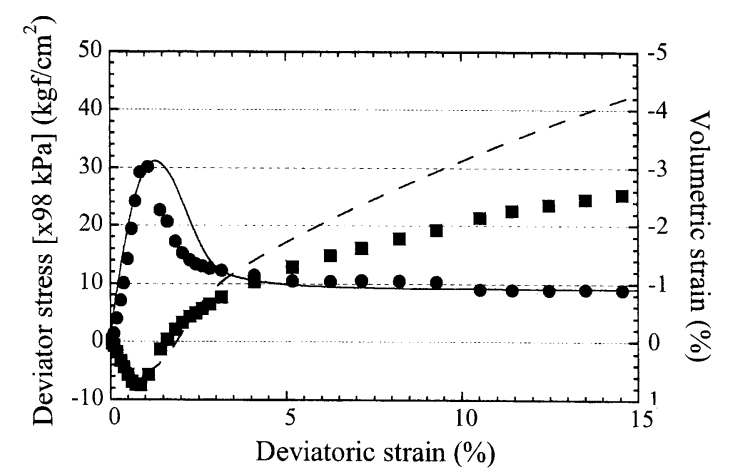

(c)

Fig. 7(c). Deviator stress-deviatoric strain and volumetric straindeviatoric strain relations (Strain rate $=\mathbf{2 . 8} \times 10^{-3} \% / \mathrm{min}$, confining pressure $98 \mathrm{kPa}$ ) 


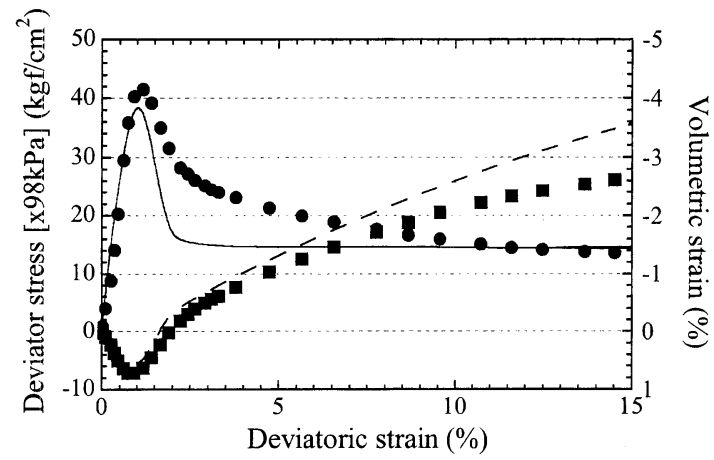

(a)

Fig. 8(a). Deviator stress-deviatoric strain and volumetric straindeviatoric strain relations (Strain rate $=\mathbf{2 . 8} \times 10^{-2} \% / \mathrm{min}$, confining pressure $294 \mathrm{kPa}$ )

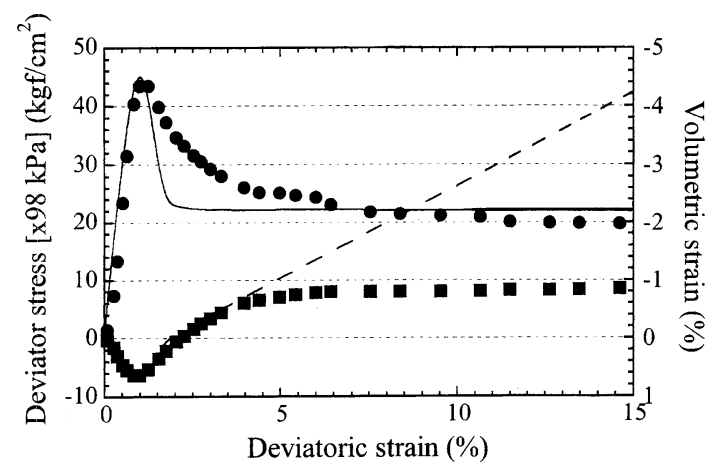

(b)

Fig. 8(b). Deviator stress-deviatoric strain and volumetric straindeviatoric strain relations (Strain rate $=2.8 \times 10^{-2} \% / \mathrm{min}$, confining pressure $490 \mathrm{kPa}$ )

The material parameters used in the calculation are in Table 2.

\section{Strain Rate Constant Triaxial Compression Tests}

Figure 7 shows a comparison between experimental and simulated results of deviator stress $\left(\sigma_{1}-\sigma_{3}\right)$-deviatoric strain $\left(e_{11}\right)$ relations. In the figure, it is seen that the proposed model well describes strain rate dependency, the peak strength and the residual strength. From Fig. 7 in which a relation between peak strength and strain rate is plotted, the proposed model can well express the effect of strain rates on the behavior. In addition, development of volumetric strain is well simulated. Figure 8 is referred to the effect of confining pressure. In this figure, the model simulation can describe the effect of confining pressure on the peak strength and the strength at large strain. The tendency, however, that the rate of strain softening with respect to strain becoming small with the increase in confining pressure is not well reproduced. The simulated results overestimate the dilatancy at large strain especially under higher confining pressure. This could be explained in the following way. In the higher confining pressure the overconsolidation boundary sur-

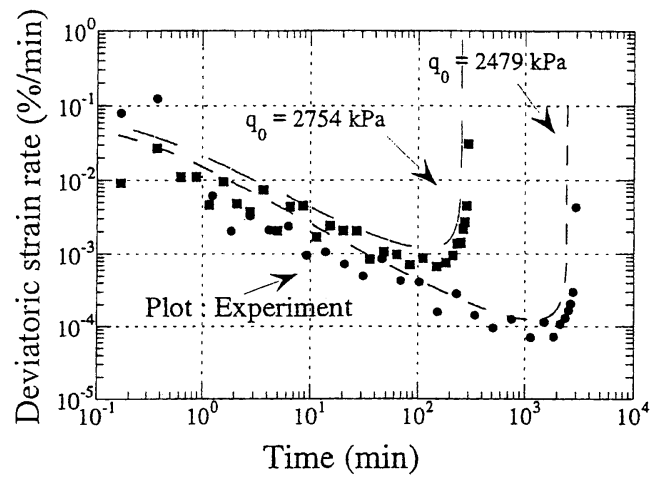

Fig. 9. Deviatoric strain rate-time relation (Drained creep, confining pressure $98 \mathrm{kPa}$ )

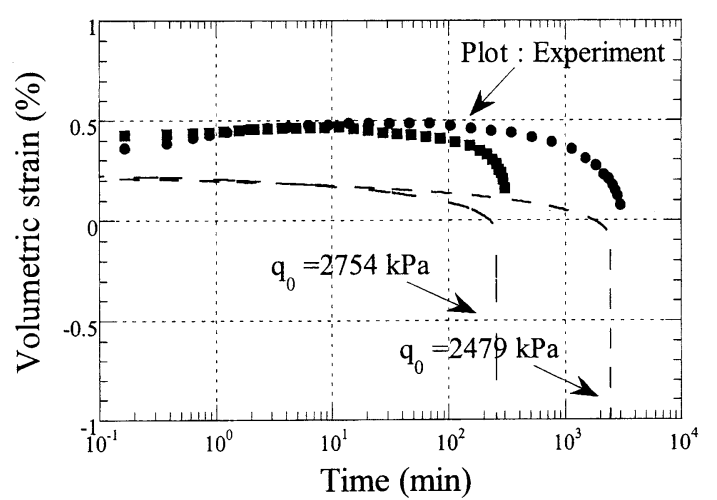

Fig. 10. Volumetric strain rate-time relation (Drained creep tests, confining pressure $98 \mathrm{kPa}$ )

face and the plastic potential surface may shrink due to the internal structural change as pointed out by Oka, Adachi and Yashima (1998). In the future this type of effect has to be incorporated in the model.

\section{Drained Creep Tests}

Figure 9 shows experimental and simulated relationship between deviatoric strain rate and time. This figure indicates that (a) the proposed model can describe the decrease in strain rate in the early stage of creep process, the minimum strain rate and the rapid increase in strain rate leading to the creep failure, and (b) acceleration of strain rate of the simulated results well correspond to the experimental results. It is well described that the creep failure time depends on the magnitude of creep load. The creep failure occurs where the magnitude of the stress is larger than the residual strength. On the other hand, when the magnitude of stress is less than the residual strength, i.e., the stress at large strain, this type of creep failure can not be attained.

Figure 10 is prepared to show the comparison between simulated and measured results of volumetric strain-time history. The significant dilatant behavior during the acceleration creep process is seen in both simulated and experimental results. Figure 11 shows a schematic diagram of stress history path in the case of drained creep 


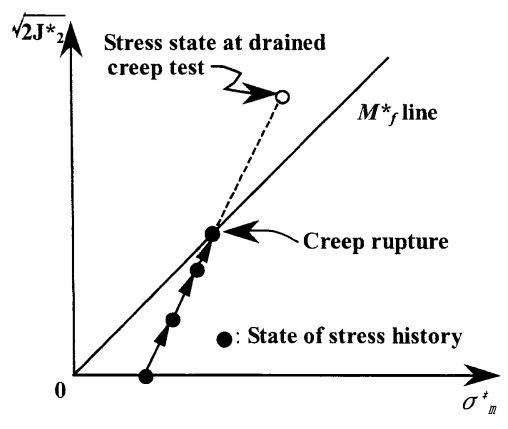

Fig. 11. Stress-history path (Drained creep test)

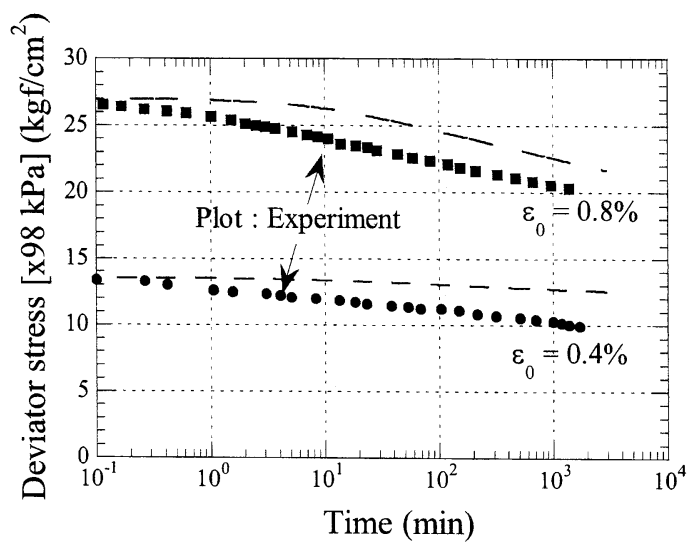

Fig. 12. Deviator stress-time relation (Undrained stress relaxation)

test. Stress history tensor $\sigma_{\mathrm{ij}}^{*}$ approaches to the real stress tensor. In the stress history space, stress history approaches the failure state monotonically although real stress increases across the failure line, and then decreases to the failure state. It is worth noting that the absorption of the pore water during drained creep conditions may occur. However, the permeability coefficient of this material has not been measured. Hence this effect cannot be estimated for the present.

\section{Undrained Stress Relaxation Tests}

Experimental and simulated deviator stress $\left(\sigma_{1}-\sigma_{3}\right)$ time profiles are shown in Fig. 12. The following two points are obtained, that is, (a) the simulated results can describe the characteristics of stress relaxation in which stress decrease is proportional to time logarithmically, and (b) in the simulated results, it is seen that the magnitude of stress relaxation depends on the value of applied stress. Larger the magnitude of applied stress, larger the rate of stress relaxation.

\section{Time Dependent Parameter $C$}

Figure 13 shows the relation between parameter $C$ and the initial mean effective stress. Considering a scattering of the data, the mean effective stress dependency on $C$ is not clearly seen. However, it might be said that $C$ is independent of the confining pressure.

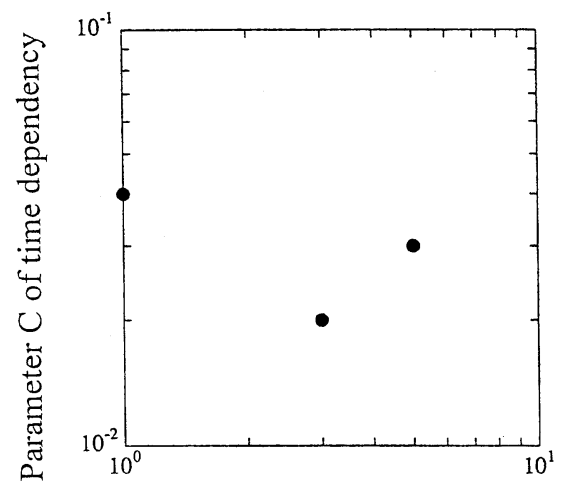

Initial mean effective stress $\sigma_{\mathrm{m} 0}^{\prime}[\mathrm{x} 98 \mathrm{kPa}]\left(\mathrm{kgf} / \mathrm{cm}^{2}\right)$

Fig. 13. Relation between time dependent parameter $C$ and initial mean effective stress $\sigma_{\mathrm{m} 0}^{\prime}$

\section{CONCLUSIONS}

An elasto-viscoplastic constitutive model for soft rocks is proposed based on the Adachi-Oka's elasto-viscoplastic constitutive model with strain softening for frozen sand. In the derivation of the model, a new definition of time measure was used and the proposed model was verified by the triaxial test results for soft rocks. It was found that the proposed model can describe the timedependent behavior such as creep, stress relaxation and strain rate constant triaxial compression tests.

(1) By introducing a new definition of time measure, an elasto-viscoplastic constitutive model for soft rock is proposed based on Adachi-Oka's elasto-viscoplastic model with strain softening for frozen sand. The proposed model can well describe the time dependent behavior such as creep, stress relaxation and strain rate constant triaxial compression test.

(2) It is found that the proposed model well simulates the stress-strain and dilatancy characteristics of Tomuro stone. Also, time dependent behavior such as strain rate sensitivity, creep and stress relaxation of Tomuro stone can be described by the model.

\section{NOTATION}

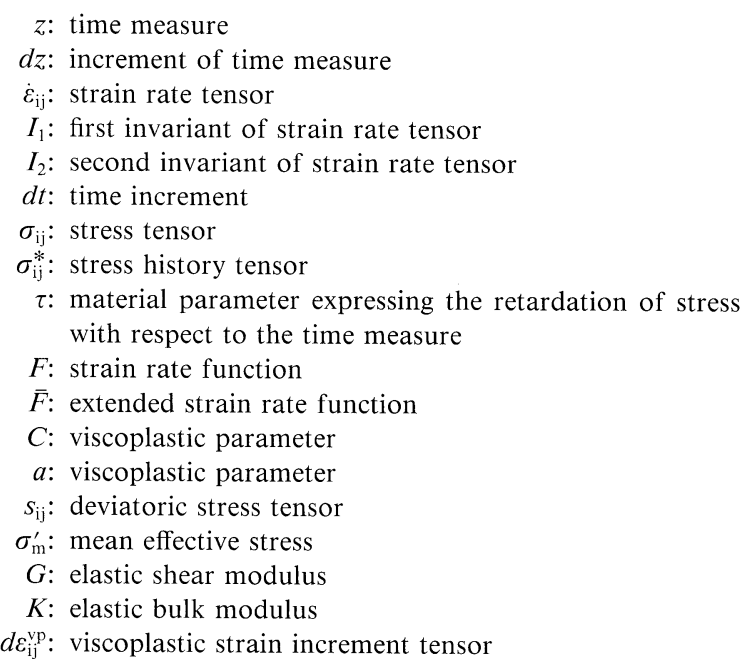


$\delta_{\mathrm{ij}}:$ Kronecker's delta

$f_{\mathrm{p}}$ : plastic potential function

$f_{\mathrm{y}}$ : plastic yield function

$\sigma_{\mathrm{m}}^{*}$ : mean stress history tensor

$S_{\mathrm{ij}}^{*}$ : deviatoric part of stress history tensor

$\eta_{i j}^{*}$ : stress history ratio tensor

$\dot{\gamma}^{\text {p: }}$ second invariant of the deviatoric plastic strain rate tensor

$\dot{e}_{\mathrm{ij}}^{\mathrm{p}}:$ deviatoric viscoplastic strain rate tensor

$\lambda_{\mathrm{v}}$ : compression index of $v-\ln \sigma_{\mathrm{m}}^{\prime}$ relation

$\kappa_{\mathrm{v}}$ : swelling index of $v-\ln \sigma_{\mathrm{m}}^{\prime}$ relation

$b$ : plastic potential parameter

$\sigma_{\mathrm{mb} 0}$ and $\bar{M}_{\mathrm{m}}$ : parameters for overconsolidation boundary surface

$M_{\mathrm{f}}^{*}$ : stress invariant ratio at failure (residual strength state)

$G^{\prime}$ : strain hardening parameter

\section{REFERENCES}

1) Adachi, T. and Oka, F. (1992): An elasto-viscoplastic constitutive model for frozen sand, Proc. Jpn. Soc. of Civil Engineers, 453(III-20), 75-81 (in Japanese).

2) Adachi, T. and Oka, F. (1995): An elasto-plastic constitutive model for soft rock with strain softening, Int. J. Numerical and Analytical Methods in Geomechanics, 19, 233-247.

3) Adachi, T., Ogawa, T. and Hayashi, M. (1981): Mechanical properties of soft rock and rock mass, Proc. 10th ICSMFE, Stockholm, Sweden, 1, 527-530.

4) Adachi, T., Oka, F. and Poorooshasb, H. B. (1990): A constitutive model for frozen sand, J. Energy Resources Technology, ASME, 112, 208-212.

5) Adachi, T., Oka, F. and Yashima, A. (1991): A finite element analysis of strain localization for soft rock using a constitutive equation with strain softening, Arch. Appl. Mech., 61, 183-191.

6) Adachi, T., Koike, M. and Oka, F. (2003): An elasto-viscoplastic constitutive model with strain softening for a sedimentary soft rock, Proc. Int. Workshop on Prediction and Simulation Methods in Geomechanics (eds. by Oka, F. et al.), Athens, JGS, 5-8.

7) Akai, K., Adachi, T. and Ando, N. (1974): Stress-strain-time relationship of saturated clay, Proc. Jpn. Soc. Civil Engineers, 225, 53-61 (in Japanese).

8) Akai, K., Adachi, T. and Ando, N. (1975): Existence of a unique stress-strain-time relation of clays, Soils and Foundations, 15(1), $1-16$.

9) Akai, K., Adachi, T. and Nishi, K. (1977): Mechanical properties of soft rocks, Proc. 9th ICSMFE, Tokyo, 1, 7-10.

10) Aversa, S., Evangelista, A., Leroueil, S. and Picarelli, L. (1993):
Some aspects of the mechanical behavior of structured soils and rocks, Proc. Int. Symp. on Hard Soils and Soft Rocks (eds. by Anagnostopoulos et al.), Athens, Balkema, 1, 359-366.

11) Cristescu, N. D. (1994): Viscoplasticity of Geomaterials, Viscoplastic Behaviour of Geomaterials, CISM, (350), Springer-Verlag, 103-207.

12) Elliot, G. M. and Brown, E. T. (1985): Yield of a soft high porosity rock, Géotechnique, 36(3), 413-423.

13) Gens, A. and Nova, R. (1993): Conceptional basis for a constitutive model for bonded soils and weak rocks, Proc. Geotechnical Engineering of Hard Soils-Soft Rocks (eds. by Anagnostopoulos et al.), Athens, Greece, Balkema, 485-494.

14) Kavvadas, M., Anagnostopoulos, A. and Kalteziotis, N. (1993): A framework for the mechanical behaviour of cemented Corinth marl, Proc. Geotechnical Engineering of Hard Soils-Soft Rocks (eds. by Anagnostopoulos et al.), Athens, Greece, Balkema, 577-583.

15) Mühlhaus, H.-B. and Aifantis, E. C. (1991): A variational principle for gradient plasticity, Int. J. Solids and Structures, 28(7), 845-857.

16) Nova, R. (1992): Mathematical modeling of natural and engineered geomaterials, Eur. J. Mechanics/A Solids, 11 (special issue), 135-154.

17) Nova, R., Castellanza, R. and Tamagnini, C. (2003): A constitutive model for bonded geomaterials subjected to mechanical and/or chemical degradation, Int. J. Numerical and Analytical Methods in Geomechanics, 27(9), 705-732.

18) Oka, F. (1985): Elasto-viscoplastic constitutive equations with memory and internal variables, Computer and Geomechanics, 1(1), 59-69.

19) Oka, F. and Adachi, T. (1985): An elasto-plastic constitutive equations of geologic materials with memory, Proc. 5th Int. Conf. on Numerical Methods in Geomechanics, Balkema, 293-300.

20) Oka, F., Adachi, T., Yashima, A. and Chu, L. L. (1994): Strain localization analysis by elasto-viscoplastic softening model for frozen sand, Int. J. Numerical and Analytical Methods in Geomechanics, 18, 813-832.

21) Oka, F., Yashima, A. and Adachi, T. (1998): A constitutive model for soft rock with both strain softening and negative dilatancy, The Geotechnics of Hard Soils-Soft Rocks, Proc. 2nd Int. Symp. on Hard Soils-Soft Rocks, (eds. by Evangelista, A. and Picarelli, L.), Naples, Italy, Balkema, 2, 737-744.

22) Valanis, K. C. (1971): A theory of viscoplasticity without a yield surface, Arch. Mech. Stos., 23(4), 515-553.

23) Valanis, K. C. (1985): On the uniqueness of solution of the initial value problem in softening materials, J. Appl. Mech., ASME, 52, 649-653. 